\title{
EDITORIAL
}

\section{Thanks to our reviewers}

\section{George Rozvany}

Published online: 25 March 2015

(C) Springer-Verlag Berlin Heidelberg 2015

On behalf of the entire editorial staff (co-editors, review editors, editorial board and Springer SMO publication and production team), I want to thank the following reviewers for their great help in maintaining and improving the quality of the journal in 2014.
N. Aage
K. Abe
E. Acar
J. Agte
D. Allaire
J. Allison
O. Amir
S. Amstutz
B. Ananthasayanam
C. Andreasen
R. Ansola
C. Antonio
S. Atamturktur
J. Atkociunas
H.-R. Bae
V. Balabanov
M. Balesdent
R. Balling
C. Barbarosie
A. Basudhar
A. Beghini
M. Berci
K. Bhachu

J. P. Blasques

S. Bobby

D. Brackett

M. Bruggi

T. Bruns

R. Burla

G. Cai

V. Challis

A. Chaudhuri

B. Chen

S. Chen

G. Cheng

C. Chevalier

J.-H. Choi

S. Choi

S. Chowdhury

A. de Faria

C.-H. Chuang

S. Degertekin

Y. Deng

Z. Dimitrovová

G. Dong

S. DorMohammadi

J. Du

Y. Du

P. Dunning

D. Durocher

P. Coelho

G. Dzierzanowski

P. Coffin

M. Eerme

I. Couckuyt

J. Enrique Rojas

P. Etman

W. Crossley

S. Ferguson

H. Dai

C. Dapogny

P. Fernandes

R. Ferreira

R. Filomeno Coelho

J. Fonseca

H. Furuya

G. Rozvany $(\bowtie)$ 

A. Gaspar-Cunha
A. Gaynor
Z. W. Geem
B. Glaz
C. Gogu
W. Gomes
J. Gravesen
A. Groenwold
D. X. Guo

S.-H. Ha

Y.D. Ha

H. Hamasaki

J. Hamel

M. Han

O. Hasancebi

W. Hoburg

B. Houska

C. $\mathrm{Hu}$

H.-Z. Huang

T. Ide
V. Jagdale
K. James
G. Jang
I. G. Jang
D. Jegley
N. Jenkins
J. Jensen
G. Jivotovski
V. Johansen
T. Johnson
S. Jung

B.-S. Kang

Z. Kang

Y. Kanno

J. Kato

M. Kazemi

G. Kennedy

K. Khandelwal

H.-G. Kim

H. Kim

H. Kim

I. Y. Kim

J. E. Kim

O. Kim

Y. Y. Kim

A. Klarbring

N. Kogiso
M. Kokkolaras

S. Koziel

A. Kumar

S. Lacaze

N. Lagaros

L. Lamberti

B. Lazarov

C. Le

L. Le Gratiet

D. Lee

E. Lee

I. Lee

J. Lee

J. Lee

L. Leifsson

$\mathrm{K}$. Lewis

M. Li

Q. Li

C. Liang

Q.Q. Liang

Q. Liao

P.T. Lin

Y. Ling

B. Liu

H. Liu

S. Liu

R. Lopez

Z. Luo

Z. Luo

H. Ma

V. Mallardo

C. Mallikarachchi

K. Marhadi

K. Marti

J. Martin

T. Martin

T. Matsumura

G. McShane

G. Michailidis

S. Missoum

Z. Mourelatos

S. Mulani

P. Nakshatrala

Y. Narita

M. Neves

T. Nguyen

E. Nikolaidis

S. Nishiwaki
B. Niu

Y. Noh

A. Novotny
M. Nowak

H. Oh

M. Ohsaki

J. Onoda

N. Pagaldipti

O. Pantz

Jo. París

C. Park

H. S. Park

Y. Park

D. Pasini

G. Paulino

C. Pedersen

N. Pedersen

V. Picheny

D. Pierre

G. Pingen

H. Pollinder

X. Qian

M. Radny

P. Ramu

T. Ray

P. Reed

G. Reich

J. Rieffel

J. Rocha de Faria

M.-il Roh

D. Romero

J. Rong

D. Rosen

M. Rumpfkeil

C. Rupp

D. Schillinger

M. Shimoda

S. Siddiqui

E. Silva

S. Singh

A. Sobester

T. Sokol

H. Song

W. Song

B. Soulier

A. Srikanth 


\section{A. Srivastava \\ B. Stanford \\ M. Stolpe \\ K. Suresh}

A. Takezawa
R. Tavakoli
A.-M. Toader

A. Todoroki

V. Toğan

M. Tootkaboni

A. Tovar

R. Troian

N. van Dijk

P. Vannucci

H. Villanueva
E. Wadbro

B. Wang

B. P. Wang

C. Wang

F. Wang

G. Wang

P. Wang

X. Wang

Z. Wang

Z. Wang

P. Wei

H.-C. Wu

Z. Xi

L. Xia

Y.-M. Xie

H. Xu
T. Yamada

J. Yan

L. Yang

S. Yang

Y. Yang

G. Yi

P. Yi

G. H. Yoon

S.-K. Youn

J. Zhang

H. Zhengdong

K. Zhou

J.-H. Zhu

P. Zhu

Z. Zhu

R. Zimmermann 